\title{
Dynamic optimization of track components to minimize rail corrugation
}

\author{
O. OYARZABAL, J. GOMEZ, J. SANTAMARIA, E. G. VADILLO \\ Department of Mechanical Engineering. University of the Basque Country UPV/EHU. Alameda \\ Urquijo s.n., 48013 Bilbao, Spain
}

NOTICE: This is an electronic version of an article published in Journal of Sound and Vibration, Vol. 319, pp. 904-917. 2009. Changes resulting from the publishing process, such as editing, corrections, structural formatting, and other quality control mechanisms may not be reflected in this document.

JOURNAL OF SOUND AND VIBRATION is available online and the final version can be obtained at:

http://dx.doi.org/10.1016/j.jsv.2008.06.020

DOI: $10.1016 / j . j s v .2008 .06 .020$

Please cite this paper as: Oyarzabal, O., Gomez, J., Santamaria, J., Vadillo, E.G. Dynamic optimization of track components to minimize rail corrugation. Journal of Sound and Vibration, Vol. 319, pp. 904-917. 2009 


\title{
Dynamic optimization of track components to minimize rail corrugation
}

\author{
O. Oyarzabal*, J. Gómez, J. Santamaría, E.G. Vadillo \\ Mechanical Engineering Department. University of the Basque Country UPV/EHU \\ Escuela Técnica Superior de Ingeniería. Alda Urquijo s/n 48013 Bilbao (Spain) \\ *Corresponding autor. Tel:+3494-601-4223 Fax:+3494-601-4215 E-mail: olatz.oyarzabal@ehu.es
}

\begin{abstract}
In this paper, the influence on corrugation of the most significant track parameters has been examined. After this parametric study, the optimization of the track parameters to minimize the undulatory wear growth has been achieved. Finally, the influence of the dispersion of the track and contact parameters on corrugation growth has been studied. A method has been developed to obtain an optimal solution of the track parameters which minimizes corrugation growth, thus ensuring that this solution remains optimum despite dispersion of track parameters and

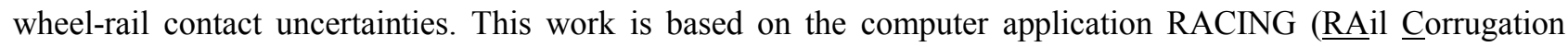
INitiation and Growth) which has been developed by the authors to predict rail corrugation features.

Keywords: rail corrugation, track dynamics, curved track, discrete support, railway vehicle, wheel-rail contact, wear

\section{Introduction}

Rail corrugation is a periodic undulatory wear that frequently appears on the rolling surface of the rail. In Fig. 1, a particular case of corrugation studied in the surroundings of Bilbao can be observed $[1,2]$. This undulatory wear provokes, apart from high dynamic loads between wheel and rail and components degradation, high levels of noise and vibrations.
\end{abstract}

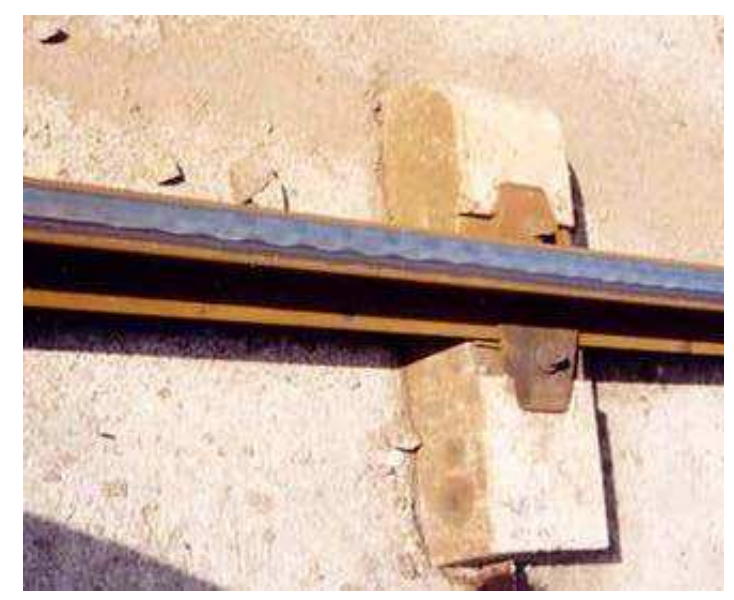

Fig. 1 Corrugation observed on the rail. 
Since the first mention of rail corrugation in scientific literature (in reference to the Cincinnati tramways, in 1895) many explanations of this problem have been published in connection with metallurgical, dynamical and tribological factors. Corrugation is still a major problem for many railway administrations, and continues to be an active field of research $[3,4,5,6]$.

Rail corrugation can generate high levels of vibrations and fast degradation of track and train components. The effects of undulatory wear can be significant, especially at high speeds, when corrugation reaches a certain amplitude. From the dynamic point of view, corrugation produces important high frequency impulses, and thus as trains move over the rails corrugation generates vibrations in the track and in the unsprung mass.

Additionally, apart from degradation of ballast and other components and high dynamic loads between wheel and rail, noise levels also increase due to corrugation. In fact, rail corrugation is one of the most serious wheel/rail contact noise problems in passenger transport systems. The noise caused by this kind of wear is irritating, and can even be harmful to the workers of a metropolitan system.

In 1993 Grassie and Kalousek published an article that reviewed 42 references and compiled the work accomplished from 1970 to 1990 [7]. In this comprehensive article corrugation is classified into six different types depending on the wear mechanism and wavelength fixing mechanism. These six types of corrugation and their wavelengths are: (1) heavy haul (200-300 mm); (2) light rail (500-1,500 mm); (3) booted sleepers (45-60 mm); (4) contact fatigue (150-450 mm); (5) rutting (50 $\mathrm{mm}$ in trams, $150-450 \mathrm{~mm}$ in trains) and (6) roaring rail (25-80 $\mathrm{mm}$ ). Although not all authors agree with this classification, the basic idea of distinguishing the two mechanisms still applies [8]. The corrugations studied in this article have elements that would fall within the booted sleepers category.

Methods to predict the appearance of corrugation have been developed [9]. Some researchers have followed a linear method for this prediction, with theories formulated in the frequency domain $[10,11,12]$. Others have developed non-linear methods based on the time domain $[13,14,15]$. These methods assume that track parameters are constant, equal to the nominal values. This paper, however, takes account of the dispersion of the track and contact parameters.

The work presented in this paper is based on a linear model developed by the authors to explain short pitch corrugation $[16,17]$. The formation of short pitch corrugation is analysed using a feedback process combining wheelset and track dynamics, contact mechanics and wear. The track and wheelset dynamics are introduced into the global model by using receptances. The track model comprised in RACING takes advantage of both Periodic 
Structure Theory and the Finite Strip Method $[18,19]$. The contact quasi-static forces and creepages are obtained through DINATREN, a theoretical model for vehicle and bogie curving developed by the authors [20]. The rail and wheel profiles are defined with spline curves. Shen, Hedrick and Elkins' contact theory has been implemented in the model [21].

Two vehicle models have been compared. The first one consists of one bogie with the yaw and lateral displacement degrees of freedom, and two wheelsets with their own yaw and lateral displacement degrees of freedom. The primary vertical, lateral and longitudinal suspensions have been taken into account. The second model consists of one whole vehicle with the two bogies previously described connected to the to the car box through the secondary suspensions. All the contact parameters have been obtained for each of the eight wheels of the first model and compared to the ones obtained for each of the four wheels of the second model. For the particular cases studied along this paper, the slight differences that appear in the contact parameters have a small significance, and do not change the corrugation results obtained. However, for other situations different to the ones analyzed in this paper (speeds, curve radii, cant, etc.), different results could be found.

Using this model a parametric study is carried out to ascertain which variables have most influence on corrugation. An optimization process then leads to the combination of track parameters which provides the lowest probability of corrugation growth. The parameters have lower and upper limits, and so optimization is developed as a problem of constrained variables. Finally, track parameter dispersion and contact uncertainties have been taken into account.

\section{Corrugation model: RACING}

In the model developed by the authors of this paper [17], an infinitesimal roughness in the profile of the rail rolling surface unleashes a feedback which generates undulatory wear on the head of the rail. This initial roughness causes infinitesimal variation of the variables which define contact between wheel and rail. Subsequently, for each excitation frequency, the variation is transmitted to the vehicle and the track, and the variation exerts its influence on dynamic behaviour.

The corrugation problem is a complex phenomenon which involves different aspects. In this model corrugation is analysed as a feedback process between the existing irregularities on the rail surface, characterised by a random wavelength; the dynamic structure of the vehicle and track; and the contact mechanics between the wheel and rail which increase wear through the existing forces in the contact. This is why the model consists of various related modules, which have been developed up to a certain degree of accuracy. 
The major modules related to the phenomenon are shown in Fig. 2. The model requires the quasi-static wheel/rail contact values obtained from the DINATREN programme while negotiating a bend. An explanation of how DINATREN works can be found in [20].

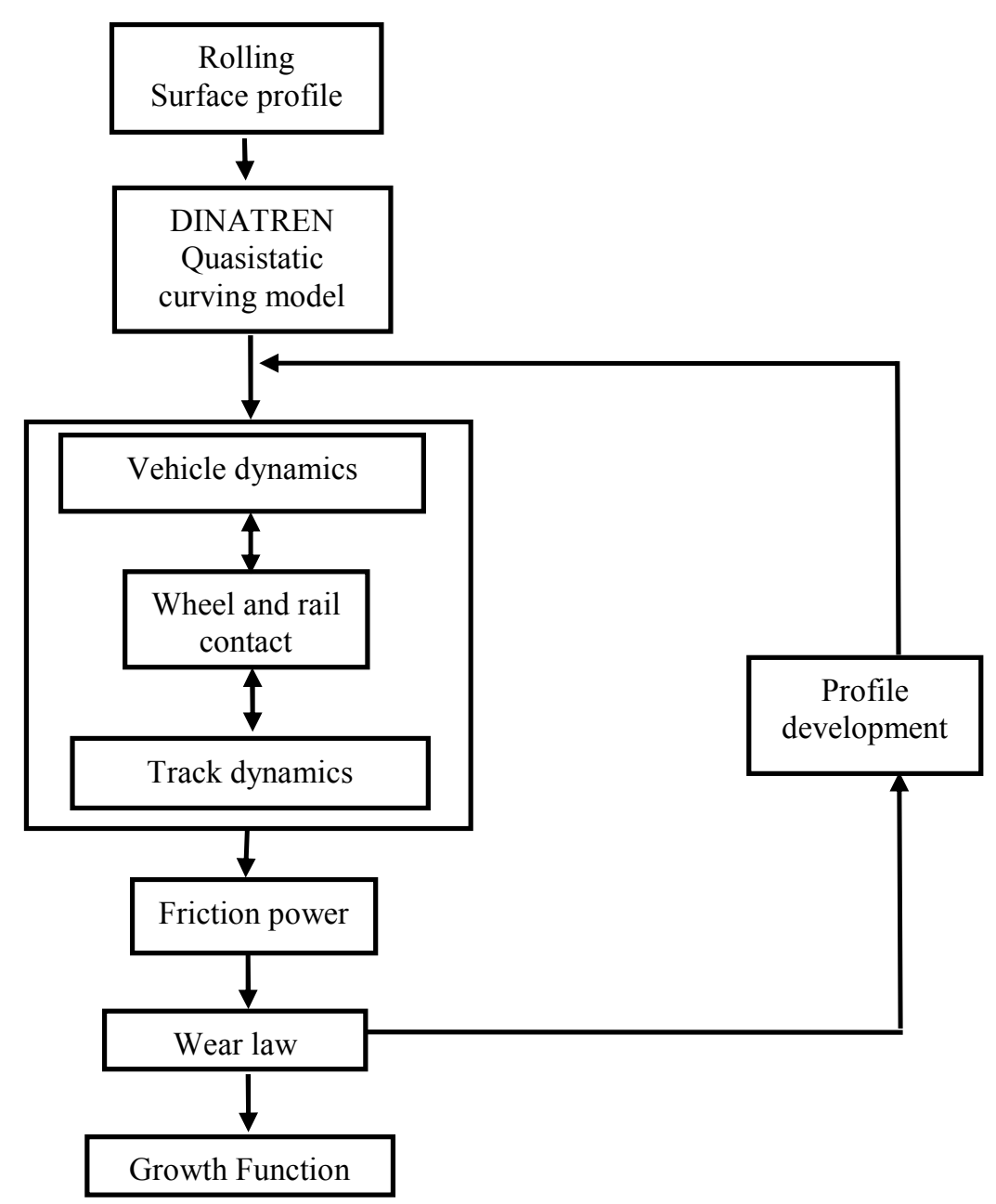

Fig. 2 Feedback cycle that generates the corrugation development.

The modular structure makes it possible to incorporate improvements in an independent way to each of the aspects contributing to corrugation. It is, moreover, possible to identify the limitations of a module and estimate its real contribution to the observed corrugation in relation to the rest of the modules. As the output of the application, the corrugation growth function, $G(f)$, provides the predisposition to appearance of corrugation depending on frequency in $\mathrm{Hz}$ [22]. RACING allows calculations of this function everywhere on the rail: at midspan, over sleeper and at every intermediate point. For this particular case the worst situation is at midspan, and for this reason most of the figures show results at midspan. Nevertheless, in Section 6 calculations are shown both at midspan and over sleeper in combination. 
The structural model to obtain the vehicle receptances consists of a 3D Finite Element model of the wheelset, brake disc and wheel boxes. The wheels have a diameter of $850 \mathrm{~mm}$.

The track model which has been used is illustrated in Fig. 3. It is important to note that the stiffness and the damping of the pad and the ballast as well as the sleeper mass are modelled in RACING in the vertical, the lateral and the longitudinal directions, although, as it is known, the longitudinal parameters have a small influence in the final results and are difficult to ascertain with accuracy. This model first obtains the free waves of the rail without support. Next, the support model is taken into account in the system, and the characteristic waves which propagate through the track with support are obtained. When the characteristic waves are calculated, the model obtains the vertical, lateral and longitudinal track receptances.

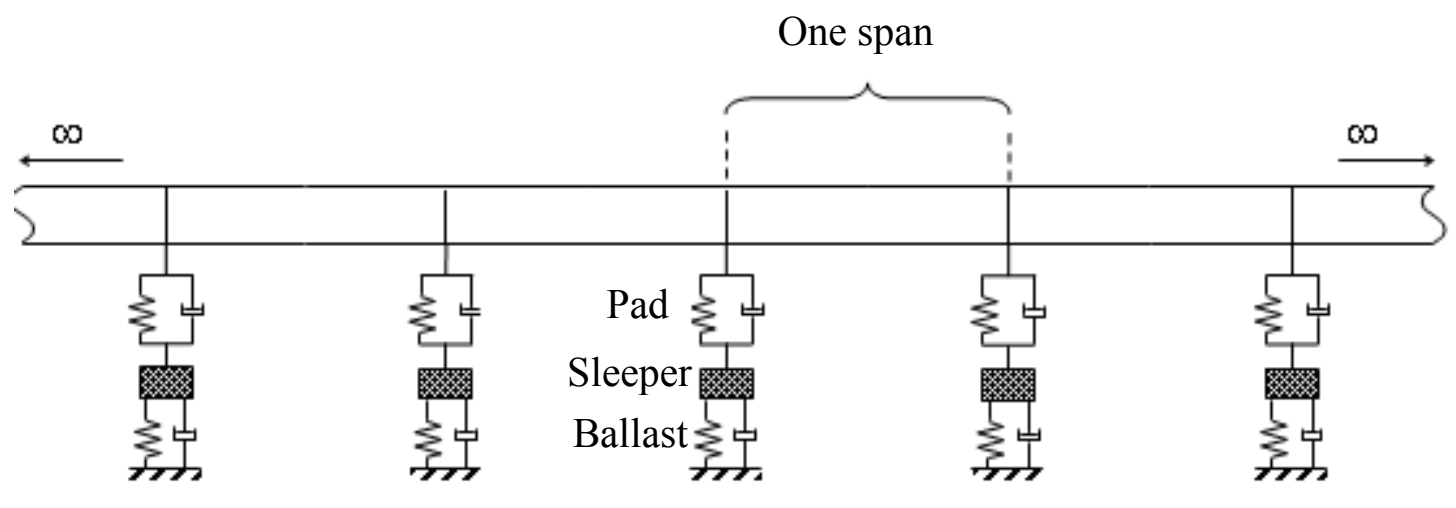

Fig. 3 Track model.

\section{Parametric study}

Recently, some authors have analysed the corrugation growth rate sensitivity to several railway parameters [23]. The current paper shows the corrugation growth functions in the frequency domain, and it has studied different parameters to those considered in the article by Meehan et al.

From the particular case of corrugation studied in Bilbao mentioned above $[1,2]$ and shown in Fig. 1, the wavelength was $62.1 \mathrm{~mm}$, the train speed was $13.8 \mathrm{~m} / \mathrm{s}$ and by implication the frequency was $222 \mathrm{~Hz}$. Corrugation grew in the inner rail and most at midspan. The curve radius was $180 \mathrm{~m}$ and the normal loads were $45.1 \mathrm{kN}$ per wheel. RACING studies the corrugation produced by each of the four wheels of the bogie. In this particular case, and using the application RACING, a parametric study has been developed. This study allows the influence on corrugation of the 14 most significant track parameters to be evaluated. These parameters are:

- Distance between sleepers, which can vary from 0.6 to $1 \mathrm{~m}$, starting with a nominal value of $1 \mathrm{~m}$.

- $\quad$ Half sleeper mass, with characteristic values between 90 and $140 \mathrm{~kg}$, starting with $96.69 \mathrm{~kg}$. 
- $\quad$ Pad stiffness and damping in longitudinal, lateral and vertical direction. The stiffness ranges from $5 \cdot 10^{7}$ to $10^{9} \mathrm{~N} / \mathrm{m}$, while the damping factor can take values between 0.1 and 0.8 . Pad vertical stiffness starting with $10^{9} \mathrm{~N} / \mathrm{m}$.

- Ballast or boot stiffness and damping in the longitudinal, lateral and vertical direction. The stiffness is of the order of $10^{8} \mathrm{~N} / \mathrm{m}$, while the damping factor can take values between 0.1 and 0.8 .

Notice that the pad stiffness high limit is rather large. It was decided to keep this value because it was obtained from the track studied in the surroundings of Bilbao.

With the parametric study it has been concluded that the five most important parameters are: distance between sleepers, sleeper mass, pad vertical stiffness, pad lateral stiffness and ballast vertical stiffness. The influence on $G(f)$ of the first, second and third parameter is shown in Figs. 4, 5 and 6. A high value of $G(f)$ at a certain frequency means a high tendency of corrugation growth at that frequency.

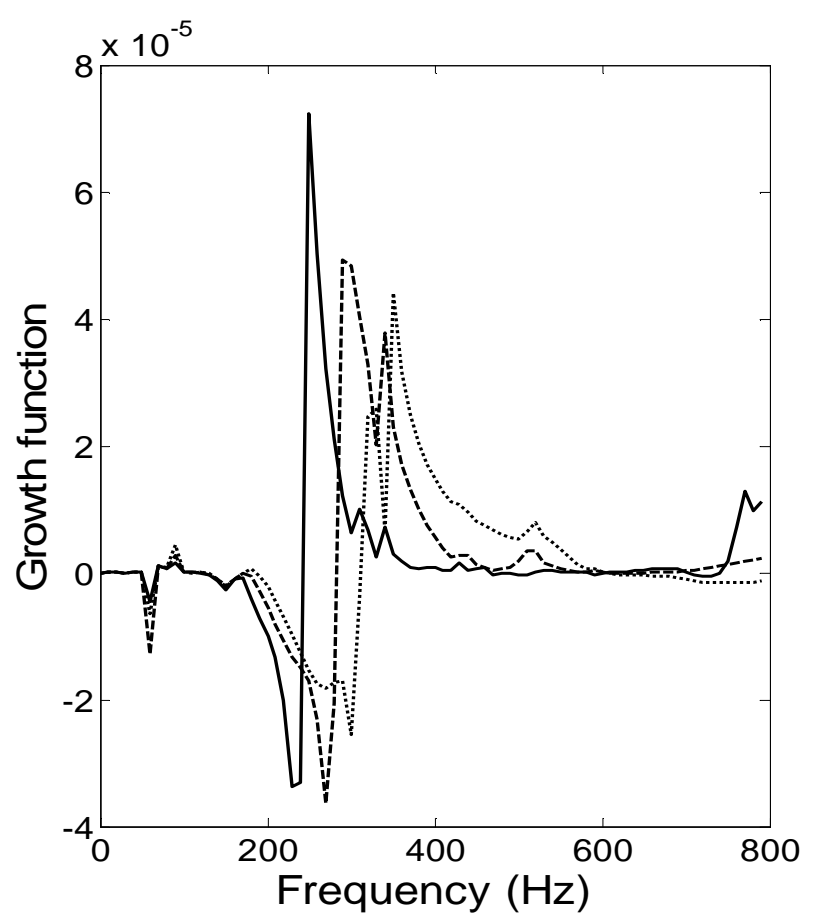

Fig. 4 The growth function at midspan for different distances between sleepers $(L) .-L=1 \mathrm{~m}$. - - $L=0.8 \mathrm{~m} . \cdots \cdots \cdots \cdots \cdots \cdots \cdots \cdots=0.6 \mathrm{~m}$.

Fig. 4 shows the growth function at midspan for different distances between sleepers. Between 200 and 400 $\mathrm{Hz}$ the maximum peak of the $G(f)$ function is different for each sleeper spacing, both in amplitude and in frequency. As it will be explained later in Section 5, this high peak is the result of a lateral resonance and a vertical antiresonance in the rail receptances. For the three values of distance between sleepers considered in the 
figure, it can be concluded that the smaller the distance between sleepers, the lower is the maximum peak of corrugation. Therefore, according to this tendency, the optimum distance between sleepers would be the smallest value, i.e. $0.6 \mathrm{~m}$. However, this is not what it is observed in the optimization study as it will be shown below. From this figure it can also be concluded that the smaller the distance between sleepers, the higher the frequency of the major corrugation peak.

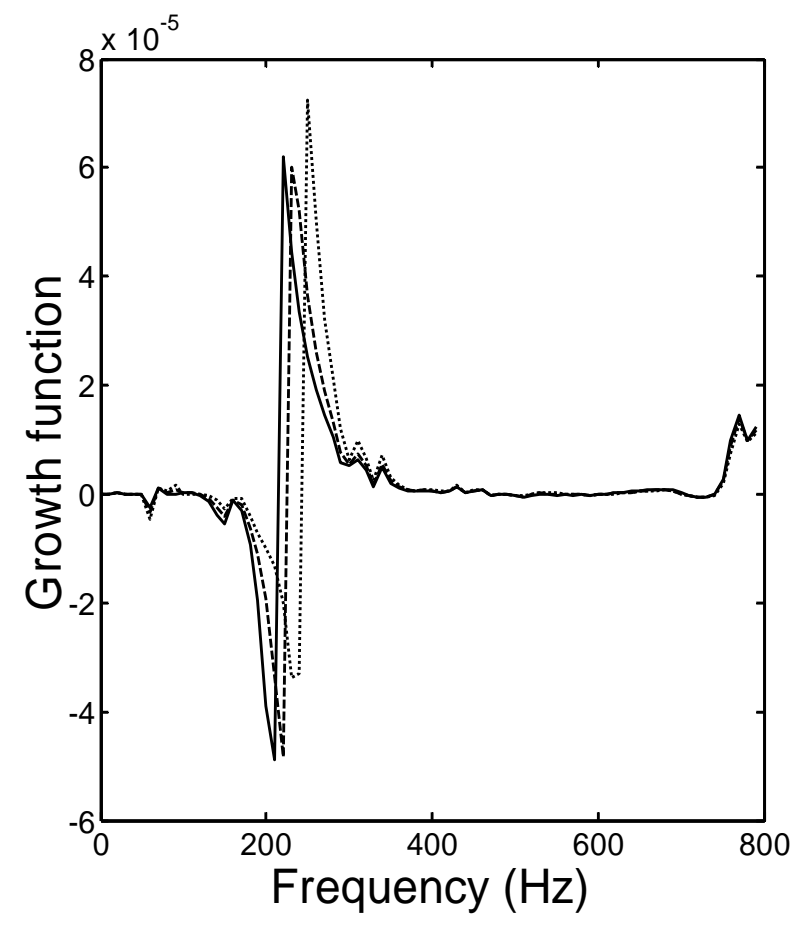

Fig. 5 The growth function at midspan for different half mass sleepers $(M s) .-M s=145 \mathrm{~kg}$. Ms $=122 \mathrm{~kg}$. ……..... $M s=96.69 \mathrm{~kg}$

Fig. 5 shows how the frequency at which the maximum peak of the growth function appears diminishes as the half sleeper mass $(M s)$ increases. It is not clear which is the optimum sleeper mass value from the parametrical study. This value has been determined in the optimization study, as will be observed in section 4.3.

In Fig. 6, the $G(f)$ function at midspan is depicted for three values of pad stiffness in the vertical direction. As it can be observed, the growth function changes notably in the $200-350 \mathrm{~Hz}$ range of frequency, when the pad stiffness in the vertical direction is changed. The lower the pad stiffness, the lower the corrugation peak becomes in these frequencies. The change in the frequency of the peak is of only $20 \mathrm{~Hz}$ from the lowest stiffness to the highest one. It is interesting to note that the distance between sleepers is $1 \mathrm{~m}$. If the span length was $0.6 \mathrm{~m}$, the variation of the peak frequency would be more significant reaching up to $80 \mathrm{~Hz}$. Above $800 \mathrm{~Hz}$, more peaks appear in $G(f)$, but due to the frequency range where usually experimentally observed corrugation takes place, these variations at high frequency are not considered in this study. 


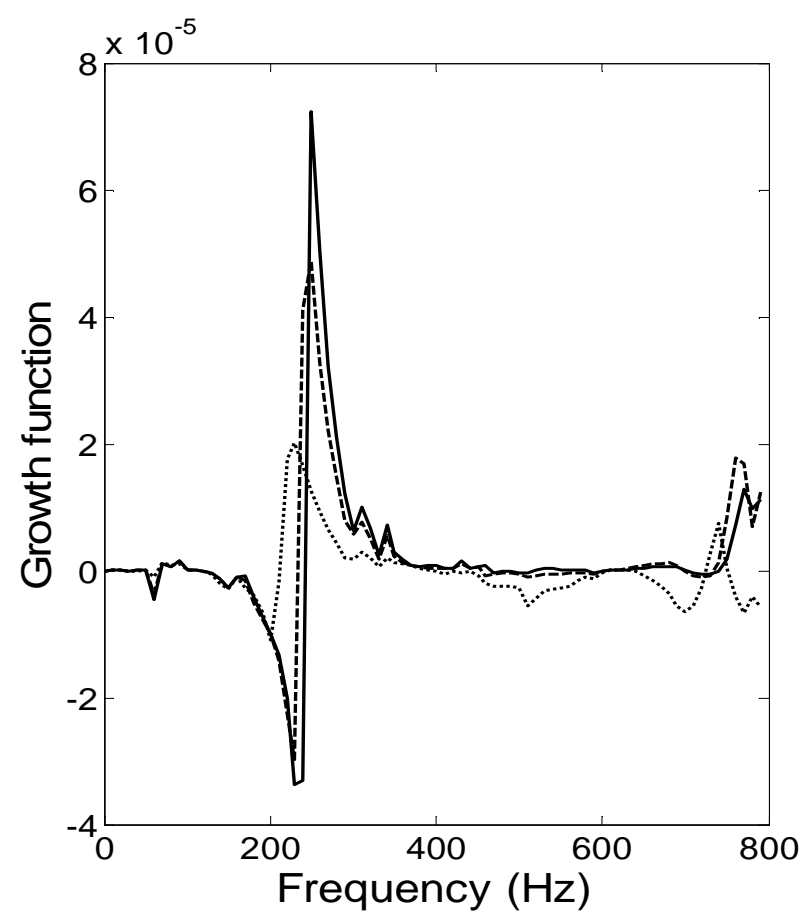

Fig. 6 The growth function at midspan for different pad vertical stiffnesses $(k z p)$. $k z p=10^{9} \mathrm{~N} / \mathrm{m}$. -... $k z p=6 \cdot 10^{8} \mathrm{~N} / \mathrm{m}$. ……........ $k z p=2 \cdot 10^{8} \mathrm{~N} / \mathrm{m}$.

\section{Optimization study}

As a conclusion of the parametric study, from the first five most important parameters, the three which have the main influence have been selected to carry out the optimization study. The optimal combination of the selected parameters that gives the smallest value for the maximum peak of $G(f)$ is now obtained.

Since RACING works in the frequency domain, the optimization process takes less computational time than using models formulated in the time domain. Moreover, since RACING avails itself of the Periodic Structure Theory and the Finite Strip Method, it is much more direct for optimization of the distance between sleepers (for example) than a model operating with finite elements in 3 dimensions. This is because optimization using such a model must overcome the linkages problem between the sleeper and the pad, and because it must maintain the aspect ratio of the $3 \mathrm{D}$ elements. If the length of the span changes, either the $3 \mathrm{D}$ elements will be distorted or it will be necessary to change the rail-sleeper linkages individually.

This model does not work in terms of pad stiffness per unit length nor sleeper mass per unit length. On the contrary it works with the pad stiffness per railseat and the half actual sleeper mass. Therefore, changes in sleeper spacing do not affect pad stiffness nor sleeper mass, making more straightforward the optimization process. 
Several frequency ranges and resolution have been used along this optimization work. However, in most cases the frequency range is from $100 \mathrm{~Hz}$ to $400 \mathrm{~Hz}$, and the frequency resolution is $5 \mathrm{~Hz}$.

\subsection{Nelder-Mead optimization}

The optimization has been performed using the Nelder-Mead method [24], a direct-search algorithm which uses function values (it does not require derivatives, so it is useful for this problem) and handles non-smooth functions. It is a numerical method for minimizing an objective function in a multi-dimensional space. The method uses the concept of a simplex, which is a polytope of $N+1$ vertices in $N$ dimensions; a line segment on a line, a triangle on a plane, a tetrahedron in three-dimensional space, and so forth. The method finds a locally optimal solution to a problem with $N$ variables when the objective function varies smoothly.

The Nelder-Mead method is implemented in Matlab [25]. The computational time required to obtain each of the optima is 36 hours, in a standard Pentium IV with 2 Gbytes of RAM memory.

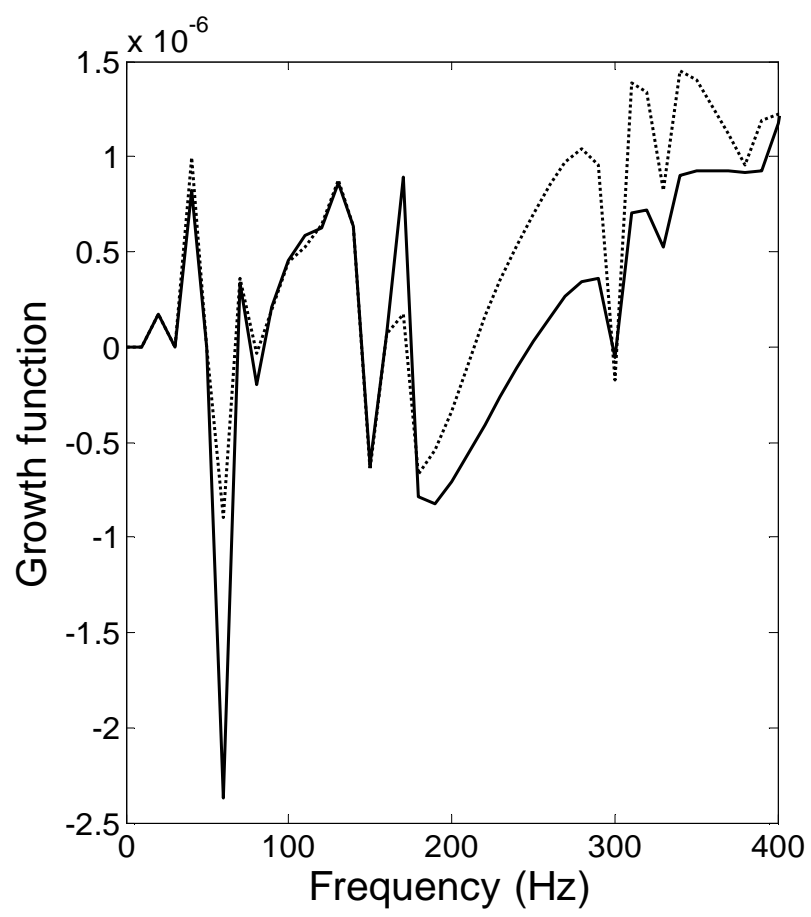

Fig. 7 The $G(f)$ furretion at midspan obtained using the Nelder-Mead method and using the Genetic Algorithm. - Genetic Algorithm --.- Nelder-Mead.

\subsection{Genetic Algorithms}

The Nelder-Mead optimization has several disadvantages, mainly that it does not explore the whole solution region and that it may find local minima. For these reasons, in this paper Genetic Algorithms (GA) have been used to find other combinations of parameters not based on a specific initial combination. The GA are very useful 
for the optimization of this problem, that has many local maxima and minima. The optimization with the genetic algorithms has proved to be more effective than with the Nelder-Mead method, as it can be seen in Fig. 7. For this particular case, the optimizations were carried out in order to obtain a $G(f)$ function in which the maximum peak between 0 and $400 \mathrm{~Hz}$ was the lowest one. As it can be seen in the figure, from 0 to $400 \mathrm{~Hz}$ and especially above $200 \mathrm{~Hz}$ the solution obtained using GA is better than the one obtained using the Nelder-Mead method.

\subsection{Results from the optimization}

The combination of the three main parameters that lead to the lowest maximum peak of $G(f)$ is considered as the optimum. Table 1 shows four optimum solutions, coming from different runs of the optimization process. Both Genetic Algorithms and the Nelder-Mead method have been used to obtain these solutions, and four important results have been selected to show them in the table, disregarding the method used to obtain them. As it can be observed in this table, the optimization study shows that three minima of the highest peak for the growth function appear when the mass of half a sleeper is around $135 \mathrm{~kg}$, and another minimum around $97 \mathrm{~kg}$.

Table 1 Combination of parameters giving the lowest maximum peak of $G(f)$.

\begin{tabular}{|c|c|c|c|c|}
\hline $\begin{array}{c}\text { Optimum } \\
\text { solution }\end{array}$ & $\begin{array}{c}\text { Distance between } \\
\text { sleepers }(\mathrm{m})\end{array}$ & $\begin{array}{c}\text { Half a sleeper mass } \\
(\mathrm{kg})\end{array}$ & $\begin{array}{c}\text { Pad vertical } \\
\text { stiffness }(\mathrm{N} / \mathrm{m})\end{array}$ & $\begin{array}{c}\text { Maximum peak of } G(f) \\
\text { function }\end{array}$ \\
\hline 1 & 0.75 & 96.72 & $5 \times 10^{7}$ & $4.03 \times 10^{-6}$ \\
\hline 2 & 0.76 & 133.32 & $5 \times 10^{7}$ & $3.32 \times 10^{-6}$ \\
\hline 3 & 0.92 & 135.35 & $7.4 \times 10^{7}$ & $3.17 \times 10^{-6}$ \\
\hline 4 & 0.99 & 138.97 & $7.8 \times 10^{7}$ & $3.13 \times 10^{-6}$ \\
\hline
\end{tabular}

The optimization study has shown that the tendencies observed in the parametric study are not valid when all the possible combinations of parameters are taken into account. Namely, the parametric study shows that the smallest distance between sleepers gives the minimum for $G(f)$. However, as it can be observed in the second column of Table 1 , none of the values is $0.6 \mathrm{~m}$. Something similar occurs for the pad vertical stiffness: the parametric study suggested that the lowest stiffness would be the optimal one, but as it can be checked in the fourth column of Table 1, two of the optimum solutions are higher than $5 \cdot 10^{7} \mathrm{~N} / \mathrm{m}$ (the lowest stiffness). It must be highlighted that those two pad stiffness are still close to the lowest one and are very soft.

Therefore, by means of the parametric study it is known which are the most important parameters, but in order to obtain the best parameter combination, the optimization study is necessary.

Another result which can be obtained from table 1 is the combination of parameters which give the lowest maximum peak of the growth function: distance between sleepers $0.99 \mathrm{~m}$; half a sleeper mass $138.97 \mathrm{~kg}$, and pad vertical stiffness $7.8 \cdot 10^{7} \mathrm{~N} / \mathrm{m}$. Besides, the other four optimum solutions are of the same order. Fig. 8 shows the 
frequency spectrum of the best and the worst optima of $G(f)$. The maximum value of the growth function is similar in both optimizations. It can be concluded from this graph that all the optima shown in Table 1 are very similar in terms of

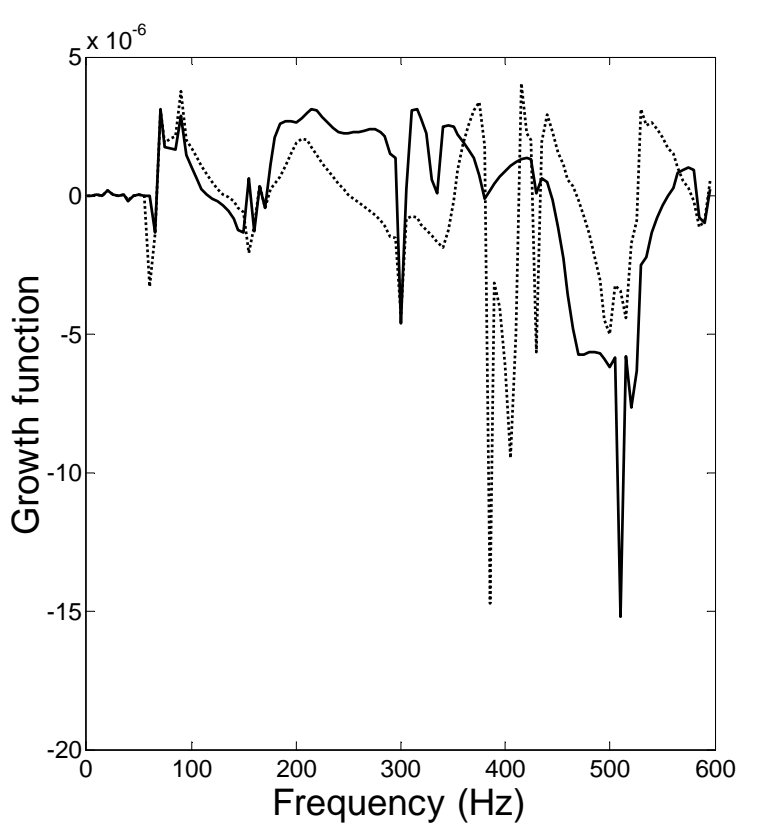
corrugation growth.

Fig. $8 G(f)$ at midspan for the best and the worst optimization. Best optimization. Worst optimization.

However, the difference in $G(f)$ between the initial data and the data that comes from the optimization is remarkable (Fig. 9). Therefore, the combination of parameters obtained from the optimization leads to an important corrugation decrease.

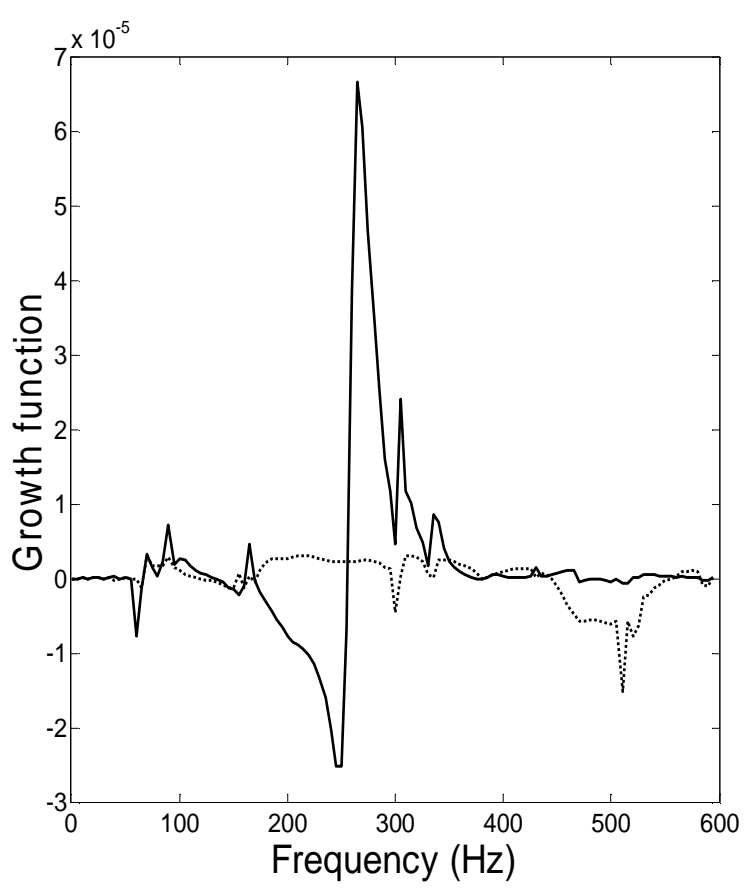

Fig. $9 G(f)$ at midspan for the initial parameters and for the best optimized parameters. Initial data Best optimization. 


\section{Track parameter dispersion and contact parameter uncertainties}

Due to the tolerances that appear during the track assembly, there is a dispersion in the track parameters and therefore in the dynamic properties. In conventional railways variations in the span length of $+/-5 \%$ are acceptable. In the sleeper mass it is necessary to distinguish between the monobloc and bibloc sleeper. Infrastructure administrations accept a tolerance in the weight of monobloc sleepers of $+/-5.5 \%$, whereas in the bibloc sleeper the upper tolerance is $4.3 \%$ and the lower tolerance is around $3 \%$ [26]. The pad stiffness in the vertical direction can vary around the nominal value by $+/-10 \%$.

These uncertainties of the track parameters lead to differences in the track receptances as can be observed in Fig. 10. If the distance between sleepers $(L)$ is increased by $5 \%$, the sleeper mass is changed by less than $5 \%$ and the pad vertical stiffness $(\mathrm{kzp})$ by $10 \%$, the track vertical and lateral receptances in midspan change significantly. It must be mentioned that these changes are applied in the model for the whole track, i.e. all pads are made stiffer or softer, etc.
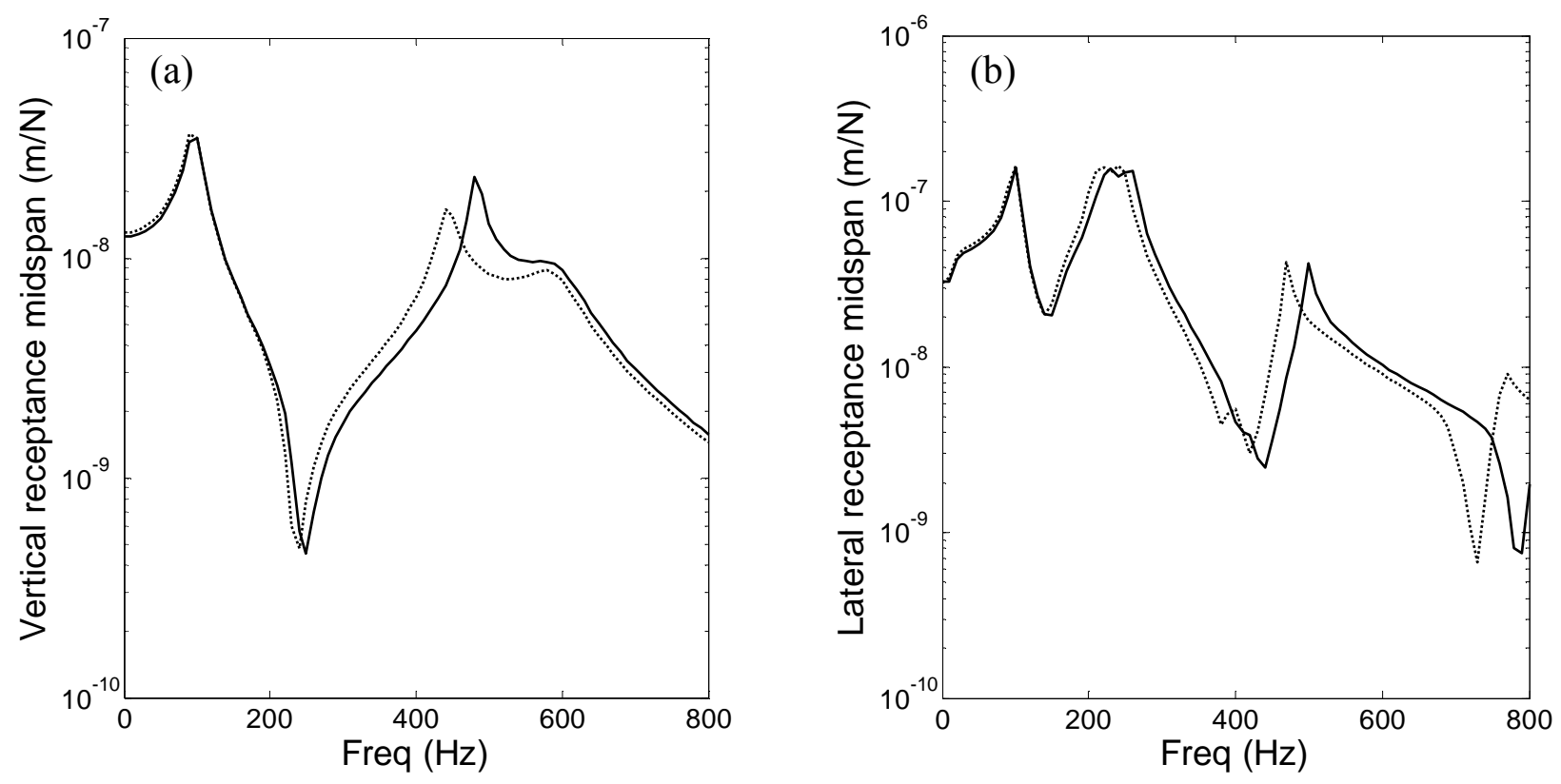

Fig. 10 Changes in track receptances, (a) vertical and (b) lateral, due to a change of $5 \%$ in distance between sleepers $(L)$, of less than $5 \%$ in the half sleeper mass $(M s)$ and of $10 \%$ in the pad vertical stiffness $(k z p) \cdots \ldots \ldots \ldots \ldots \ldots \ldots=1$ $\mathrm{m}, M s=96.69 \mathrm{~kg}, k z p=10^{9} \mathrm{~N} / \mathrm{m} ; \quad L=0.95 \mathrm{~m}, M s=95.5 \mathrm{~kg}, k z p=9 \cdot 10^{8} \mathrm{~N} / \mathrm{m}$

In Fig. 10, the peak in the vertical receptance at midspan which appears at around $450 \mathrm{~Hz}$ is due to the pinned-pinned mode. The peak that appears in the same graph at around $100 \mathrm{~Hz}$ is due to the ballast. Finally, the antirresonance obtained at around $230 \mathrm{~Hz}$ in vertical receptance and the corresponding peak at approximately the 
same frequency in lateral receptance, due to the lateral pinned-pinned, work together to give the maximum peak in the corrugation growth function according to RACING. This can be checked by observing figures 4 to 6 and noting how the highest peak of the growth function (for $\mathrm{L}=1 \mathrm{~m}, \mathrm{Ms}=96.69 \mathrm{~kg}$ and $\mathrm{kzp}=10^{9} \mathrm{~N} / \mathrm{m}$ ) appears around that frequency of $230 \mathrm{~Hz}$.

Variations in train speeds give rise to uncertainties in the contact parameters of each wheel. There have been estimated changes of $10 \%$ in the semi-axes of the contact ellipse, in the lateral and longitudinal creepages and in the contact normal forces. This estimation is based on calculations made with the program DINATREN to obtain the contact values while negotiating a bend [20].

As has been shown, the track parameters have a considerable dispersion which significantly influences track receptances, and there are also major uncertainties in the contact parameters. Therefore, the corrugation growth tendencies are affected. Thus, although there is always a theoretical optimum solution, small changes in the track and in the contact values due to this dispersion could introduce an important peak in the growth function, and this would signify a tendency towards corrugation growth (Fig. 11). In this figure a major increase in the growth function may be observed above $600 \mathrm{~Hz}$, as a consequence of the change of the parameters shown in table 2 . These changes are less than the abovementioned acceptable limits of $+/-5 \%$ and $+/-10 \%$ in the track and contact parameters.

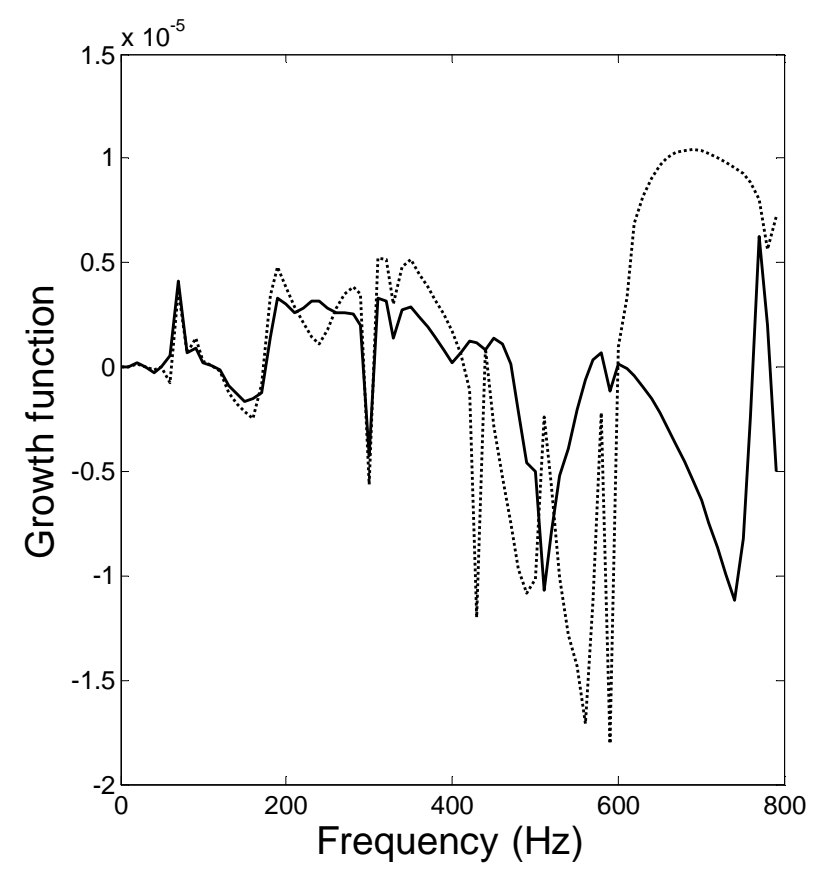

Fig. 11 Important change in the growth function due to dispersion of the contact and track parameters.

Optimum.

Next to optimum. 
Table 2. Combination of parameters next to the optimum that cause an important change in the growth function

\begin{tabular}{|c|c|c|c|c|c|c|c|c|}
\cline { 2 - 8 } \multicolumn{1}{c|}{} & \multicolumn{3}{c|}{ Track parameters } & \multicolumn{5}{c|}{ Contact parameters } \\
\hline & $\begin{array}{c}\text { Distance } \\
\text { between } \\
\text { Sleepers } \\
(\mathrm{m})\end{array}$ & $\begin{array}{c}\text { Sleeper } \\
\text { Mass } \\
(\mathrm{Kg})\end{array}$ & $\begin{array}{c}\text { Pad } \\
\text { Vertical } \\
\text { Stiffness } \\
(\mathrm{N} / \mathrm{m})\end{array}$ & $\begin{array}{c}\text { Semi-axes } \\
\mathrm{a}(\mathrm{mm})\end{array}$ & $\begin{array}{c}\text { Semi-axes } \\
\mathrm{b}(\mathrm{mm})\end{array}$ & $\begin{array}{c}\text { Normal } \\
\text { Force } \\
(\mathrm{N})\end{array}$ & $\begin{array}{c}\text { Lateral } \\
\text { creepage }\end{array}$ & $\begin{array}{c}\text { Longitudinal } \\
\text { creepage }\end{array}$ \\
\hline Optimum & 0.96 & 95 & $7.81 \times 10^{7}$ & 5.27 & 4.19 & 44929 & -0.0084 & 0.0055 \\
\hline $\begin{array}{c}\text { Next to } \\
\text { optimum }\end{array}$ & 0.91 & 96.5 & $7.42 \times 10^{7}$ & 4.74 & 4.39 & 49420 & -0.0087 & 0.0050 \\
\hline
\end{tabular}

To overcome this problem a method has been developed to find an optimum of the growth function which remains an optimum when the track and contact parameters change slightly due to the abovementioned dispersion. The solution to the problem may be observed in the flow chart shown in Fig. 12:

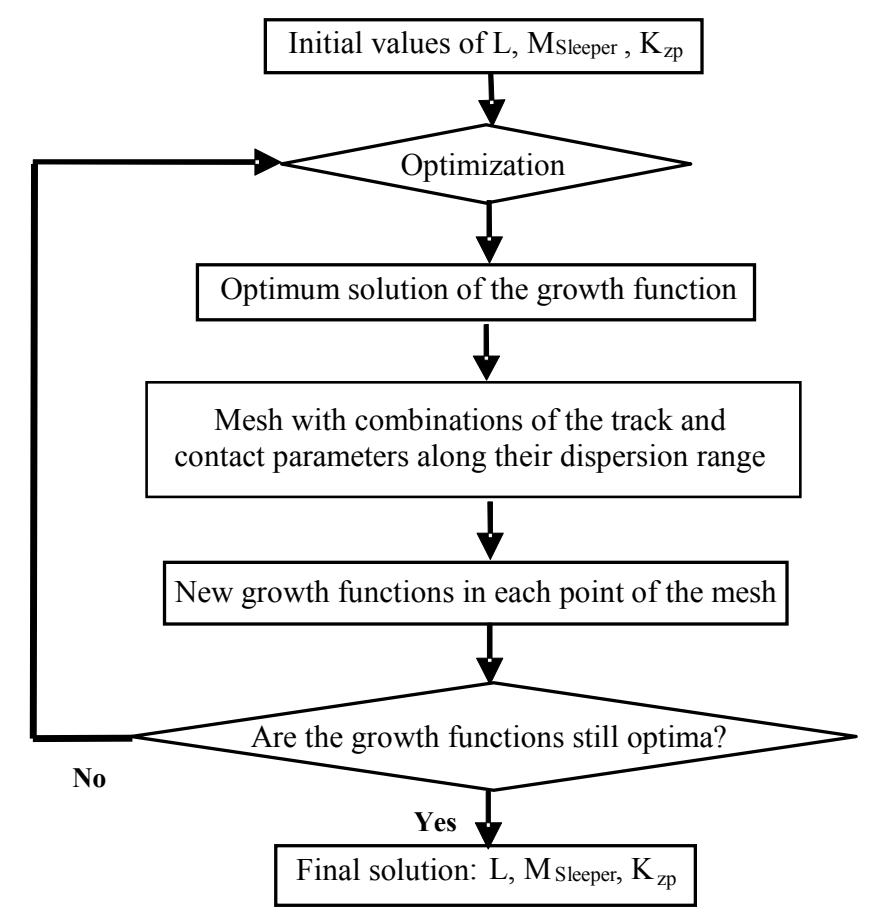

Fig. 12 Method developed to prevent a high growth function for a combination of parameters close to those of an optimum solution.

- The programme starts with the initial values of the distance between sleepers $(L)$, the sleeper mass $\left(M_{\text {sleeper }}\right)$ and the pad vertical stiffness $(k z p)$. An optimization process is carried out, and a first optimum solution of the growth function is obtained, together with the values of $L, M_{\text {sleeper }}$ and $k z p$ that lead to it. 
- Each of the track and main contact parameters is discretized along their dispersion ranges, and a mesh is created with all the combinations of discretized parameters. The growth function is calculated for each combination, and it is checked if any of these new growth functions has high peaks. The computation time required for this step is very low, compared to that of the optimization process.

-If none of these new growth functions have high peaks, or if all these functions are extremely plain, the optimum solution values of track parameters $L, M_{\text {sleeper }}$ and $k z p$ that had been obtained are accepted as the final solution. These values will remain optimum despite any possible changes in the dispersion range of the track and contact parameters.

- However, if one or more of these new growth functions have high peaks, it is necessary to run again the optimization process. The GA optimization will lead, in general, to an optimum solution different from the one obtained the previous time in step 1. Steps 2 to 4 are repeated until the growth functions of all the combinations of track parameters (centred around the optimum solution) and of contact parameters, discretized along their dispersion ranges, remain plain.

\section{Optimum at midspan and above a sleeper}

Before a new line project is accomplished, or whenever a corrugation problem needs to be solved, it is necessary to ascertain a combination of track parameters leading to an optimum of the growth function both above a sleeper and at midspan. For this purpose, an auxiliary function $A(f)$ has been used which is the sum of the positive values of the growth functions at midspan and over a sleeper.

As may be observed in Fig. 13 at midspan (left), optimization was extremely good before using the auxiliary function, but above a sleeper (right) there was a peak which disappears when optimized using the auxiliary function, $\mathrm{A}(\mathrm{f})$, and taking into account both midspan and above a sleeper. 

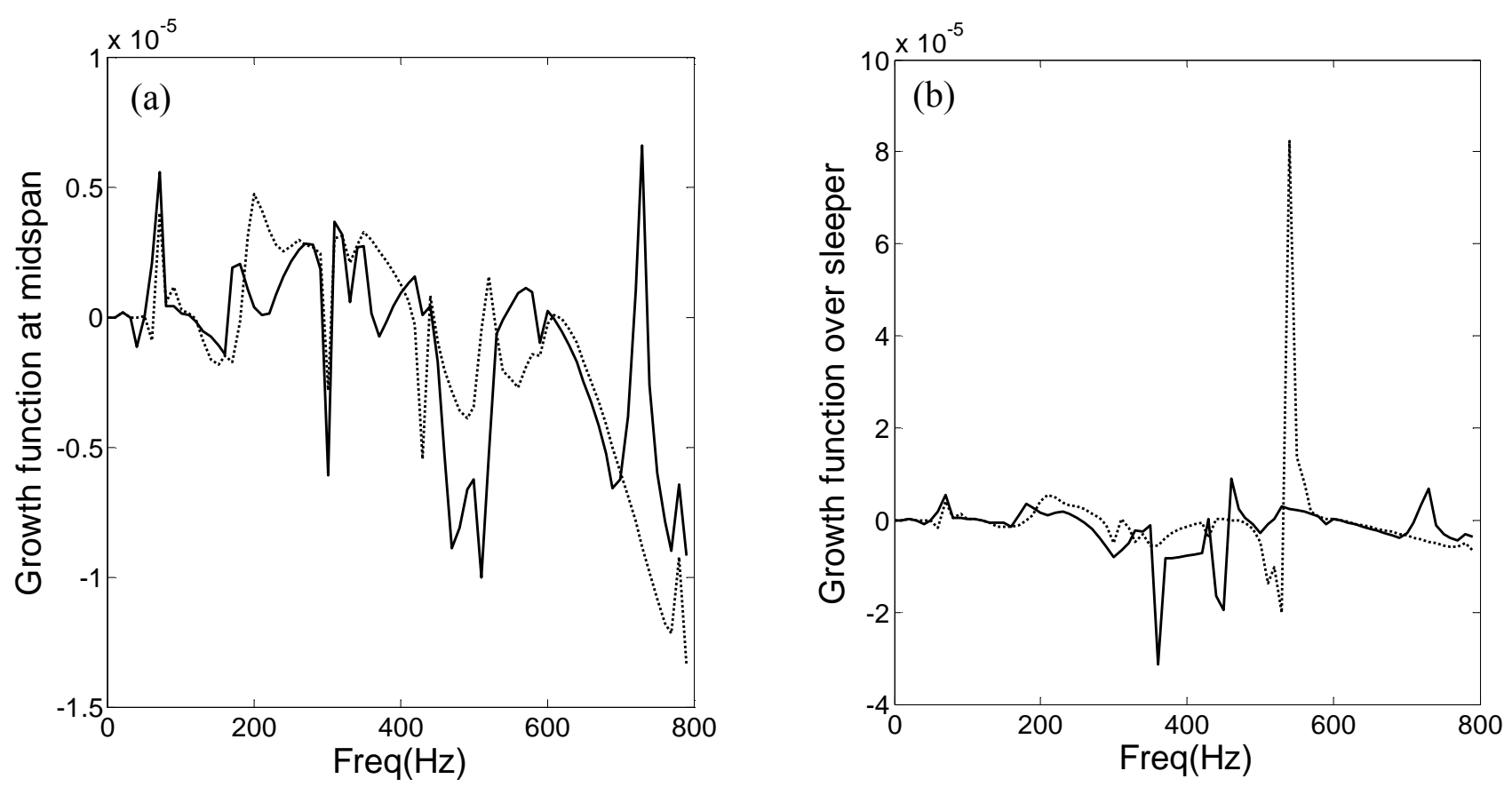

Fig. 13 Optimized growth function at midspan (a) and over sleeper (b), for a combination of track parameters that optimize corrugation at midspan compared to global optimization at both midspan and sleeper, A(f).

Optimized at midspan. $\quad$ Optimized both above a sleeper and at midspan, using the auxiliary function A(f).

\section{Conclusions}

In this paper a computational application developed by the authors (RACING) has been used to study the corrugation growth. This application obtains track and wheelset receptances and considers corrugation as a complex feedback determined also by contact parameters.

The tool developed has been used to carry out a parametric study in order to find the influence of the 14 main parameters. Among the track parameters, the following five have more influence on the corrugation: distance between sleepers, sleeper mass, pad vertical stiffness, pad lateral stiffness and ballast vertical stiffness. The optimization study has been carried out focusing the attention on the first three parameters.

From the optimization work, combinations of parameters are obtained which give an optimum $G(f)$ function, this is, the lowest maximum peak of $G(f)$. Two optimization techniques have been used to obtain a combination of parameters which lead to an optimum in the growth function. Optimization using Genetic Algorithms has proved to be more effective than using the Nelder-Mead method. The improvement obtained with the optimization is very significant.

It has been considered that the distance between sleepers as well as the sleeper mass and the pad vertical stiffness have a physical dispersion that cannot be neglected. Because of this dispersion, changes appear in the 
track receptances. Taking this dispersion into account, and also the uncertainties in the contact parameters, changes appear in the corrugation growth function.

A method has been developed to obtain an optimal solution of the track parameters which minimizes corrugation growth, making sure that this solution remains an optimum, taking into account the track parameters dispersion and the uncertainties of the contact parameters.

Using the auxiliary function $\mathrm{A}(\mathrm{f})$ it is possible to complete global optimization at both midspan and sleeper.

\section{Acknowledgements}

The authors are grateful to CEDEX for their support through contract PT2006-024-19CCPM. The authors also acknowledge the financial help received from the Department of Education, Universities and Research of the Basque Government.

\section{References}

[1] E.G. Vadillo, J.A. Tárrago, G. Gárate, C. Angulo, Effect of sleeper distance on rail corrugation. Wear 217 (1998) $140-146$.

[2] I. Gómez, E.G. Vadillo, An analytical approach to study a special case of booted sleeper track rail corrugation, Wear 251 (2001) 916-924.

[3] Y. Suda, M. Hanawa, M. Okumura, T. Iwasa, Study on rail corrugation in sharp curves of commuter line Wear 253 (1-2) (2002) 193-198

[4] T.X. Wu, D.J. Thompson, An investigation into rail corrugation due to micro-slip under multiple wheel/rail interactions Wear 258 (7-8) (2005) 1115-1125

[5] X. Sheng, D.J. Thompson, C.J.C. Jones, G. Xie, S.D. Iwnicki, P. Allen and S.S. Hsu, Simulations of roughness initiation and growth on railway rails. Journal of Sound and Vibration 293, Issues 3-5, (2006) Pages 819-829

[6] X.S. Jin, Z.F. Wen, K.Y. Wang, Z.R. Zhou, Q.Y. Liu and C.H. Li, Three-dimensional train-track model for study of rail corrugation. Journal of Sound and Vibration 293, Issues 3-5 (2006) 830-855.

[7] S.L. Grassie, J. Kalousek, Rail corrugation: characteristic, causes and treatments, Proc. Inst. Mech. Eng. 207 (1993) 57-68.

[8] S.L. Grassie, Rail corrugation: advances in measurement, understanding and treatment, Wear 258 (2005) 1224-1234.

[9] Yoshihiko Sato, Akira Matsumoto and Klaus Knothe, Review on rail corrugation studies Wear 253 (1-2) (2002) $130-139$.

[10] E. Tassilly, N. Vincent, A linear model for the corrugation of rails, Journal of Sound and Vibration 150 (1) (1991) 25-45.

[11] Hempelmann, K., Short pitch corrugation on railway rails. A linear model for prediction, VDI-Fortschrittberichte, number Reihe 12, Nr. 155. VDI-Verlag, Düseldorf (1994).

[12] S. Müller, A linear wheel-rail model to investigate stability and corrugation on straight track, Wear 243 (1-2) (2000)122-132 
[13] J .C. O. Nielsen, A. Igeland, Vertical dynamic interaction between train and track- Influence of wheel and track imperfections, Journal of Sound and Vibration 187 (5) (1995) 825-839

[14] H. Ilias, The influence of railpad stiffness on whelset/track interaction and corrugation growth, Journal of Sound and Vibration 227 (5), (1999) 935-948

[15] C. Andersson, A. Johansson, Prediction of rail corrugation generated by three-dimensional wheel-rail interaction Wear 257 (34), (2004) 423-434

[16] I. Gómez, E.G. Vadillo, A linear model to explain short pitch corrugation on rails, Wear 255 (2003) 1127-1142.

[17] J. Gómez, E.G. Vadillo, J. Santamaría, A comprehensive track model for the improvement of corrugation models Journal of Sound and Vibration 293, Issues 3-5 (2006) 522-534.

[18] L. Gry, Dynamic modelling of railway track based on wave propagation, Journal of Sound and Vibration 195 (3), (1996), $477-$ 505.

[19] L. Gry, C. Gontier, Dynamic modelling of railway track: a periodic model based on a generalized beam formulation, Journal of Sound and Vibration 199 (4) (1997), 531-558.

[20] J. Santamaría, E.G. Vadillo, J. Gómez, A comprehensive method for the elastic calculation of the two-point wheel-rail contact, Vehicle System Dynamics 44 (supl) (2006), 240-250.

[21] Z.Y. Shen, J.K. Hedrick, J.A. Elkins, A comparison of alternative creep force models for railway vehicle dynamic analysis, Vehicle System Dynamics 12 (1-3) (1983), 79-82.

[22] C. O. Frederick, A rail corrugation theory, Proceedings of the International Symposium on the Contact Mechanics and Wear of Rail/Wheel systems, Kingston, Rhode Island (1986) 181-211.

[23] Meehan P.A., Daniel W.J.T., Campey T., Prediction of the growth of the wear-type growth corrugation, Wear 258 (2005), 10011013.

[24] Reklaitis G. V., Ravindran A., Ragsdell K. M., Engineering Optimization: Methods and Applications, John Wiley and Sons, New York (1983)

[25] MATLAB 7.2. User's manual, The Mathworks. Inc, Natick, MA, 2006.

[26] Euskotren, Provision and acceptance of bibloc sleepers for STEDEF slab track (In Spanish) EV-8-EV-1-003. November 2000 